\title{
¿Por qué los profesionales prefieren las redes sociales académicas antes que los repositorios a la hora de difundir sus publicaciones?
}

José Javier García Vicente | Portal Todopatrimonio.com

URL de la contribución <www.iaph.es/revistaph/index.php/revistaph/article/view/4650>

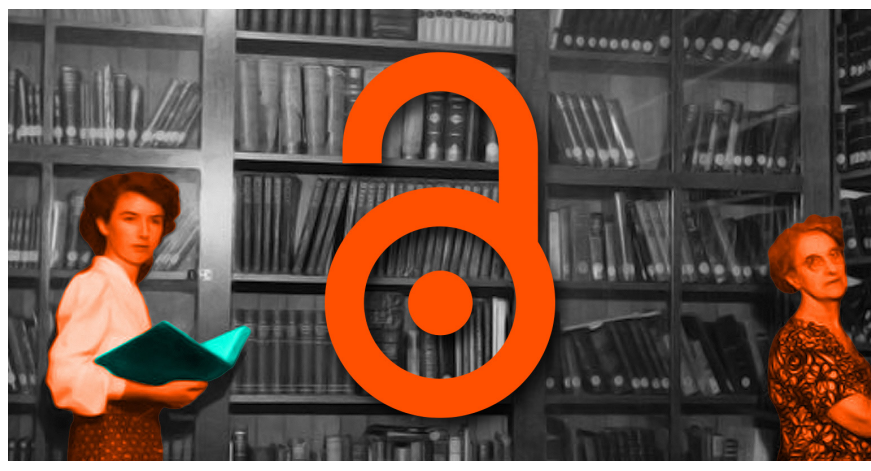

Durante la semana del Acceso Abierto digamos juntos \#YoComparto | foto Fundación Karisma Colombia

La evolución de las tecnologías de la información y de internet está facilitando a los profesionales no sólo el acceso a una ingente cantidad de documentación y literatura científica, sino que además les proporciona una serie de herramientas para ayudarles a difundir su propia producción científica. Para conseguir el acceso abierto a la documentación científica y técnica los investigadores pueden optar por dos vías: a) la conocida como vía verde, que no es otra cosa que depositar los artículos ya publicados y otras publicaciones en algún repositorio; b) la vía dorada, que consiste en publicar en revistas en acceso abierto.

En lo que respecta a la vía verde, los profesionales tienen la opción de depositar sus publicaciones en el repositorio de la institución donde trabajan o, en su defecto, en algún otro repositorio temático. En las dos últimas décadas son numerosas las instituciones, y principalmente las universidades, que han implementado un repositorio institucional para aglutinar, almacenar, difundir y preservar a largo plazo todo el acervo documental pro- ducido por sus investigadores a lo largo de su historia. Según muestra el directorio de repositorios OpenDOAR, a fecha de abril de 2020 había implementados más de 5300 repositorios en todo el mundo, tanto disciplinarios, multidisciplinarios, como institucionales. Los profesionales tienen por consiguiente la posibilidad de depositar sus publicaciones en alguno de estos repositorios para proporcionar más difusión, visibilidad y accesibilidad a su producción científica.

Muchas de las instituciones que han implementado un repositorio han adoptado igualmente una política de depósito para ayudar e incentivar a sus investigadores a depositar las publicaciones en él.

A pesar de las ventajas y beneficios que ofrecen los repositorios para los autores en cuanto a visibilidad y difusión, la comunidad científica parece preferir difundir las publicaciones a través de las redes sociales académicas (Academia.edu o ResearchGate) antes que en aquellos. Hay ya varios estudios al respecto que muestran esta preferencia de los científicos por las redes sociales. En 2017, el profesor Ángel Borrego, de la Universitat de Barcelona, publicaba en un artículo los resultados de un estudio en el que comparaba la producción científica de 13 universidades españolas y su disponibilidad tanto en sus respectivos repositorios institucionales como en ResearchGate. El estudio mostraba que únicamente el $11.1 \%$ de los artículos publicados eran depositados en el repositorio de la universidad, frente al $54.8 \%$ que se encontraban en ResearchGate.

Desde el portal Todopatrimonio.com hemos llevado a cabo una pequeña encuesta para conocer el compor- 
a debate Repositorios y redes sociales académicas para la transferencia del conocimiento abierto

| coordina Remedios Melero Melero

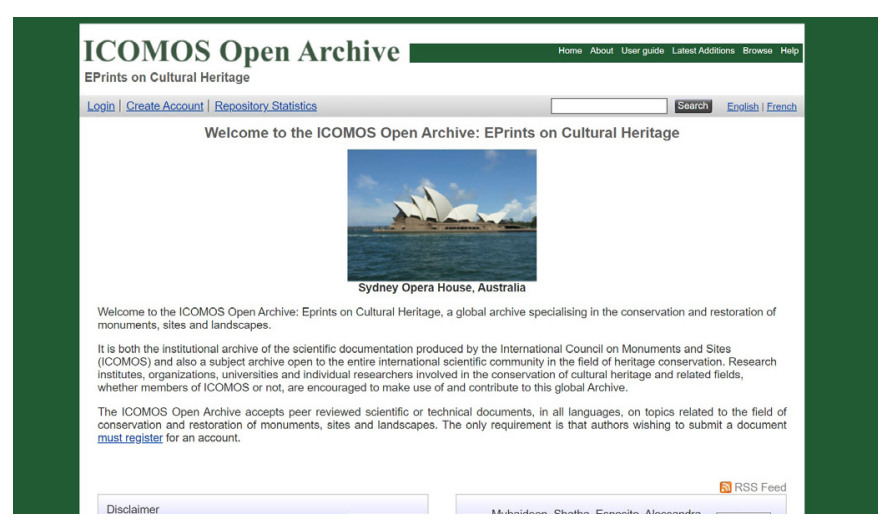

ICOMOS Open Archive

tamiento de los profesionales del patrimonio en lo que respecta al uso de repositorios y redes sociales académicas. Los profesionales que han respondido a nuestro estudio se encuentran dentro del ámbito iberoamericano, habiendo recibido respuestas provenientes de personas de 13 países.

Entre las opciones propuestas, el 65\% de los encuestados afirma difundir sus publicaciones científicas en redes sociales académicas, frente al 39\% que lo hace en los repositorios. En cuanto a las redes sociales se refiere, muchos profesionales difunden sus publicaciones en varias redes, siendo la preferida con diferencia Academia.edu (67\%) frente a ResearchGate (29\%) y Linkedin (29\%). Mendeley únicamente es utilizada por el $8 \%$.

Entre aquellos profesionales que depositan sus artículos científicos en los repositorios, la inmensa mayoría indican que suele hacerlo únicamente en uno, generalmente en el de sus universidades respectivas. El 10\% en cambio afirma depositar sus trabajos en el ICOMOS Open Archive.

Las respuestas obtenidas a varias de las preguntas que planteábamos nos muestran en primer lugar un desconocimiento general sobre la existencia de repositorios y su funcionamiento. En algunos casos se produce una confusión entre estos y las redes sociales académicas, no sabiendo diferenciar a unos de otras. Ante la pregunta

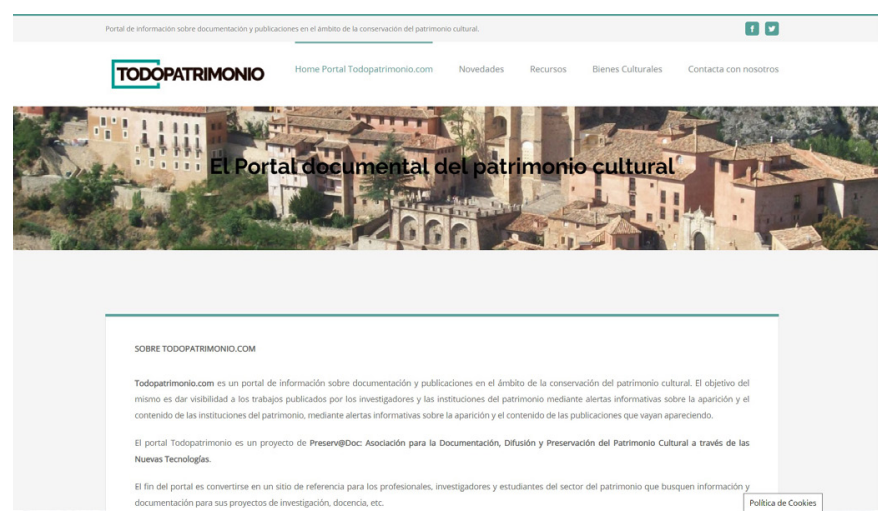

Portal Todopatrimonio

sobre el grado de dificultad para depositar en repositorios o redes sociales, la inmensa mayoría considera que el proceso para realizar el depósito es mucho más sencillo en las redes sociales que en los repositorios. Sólo el $5 \%$ prefería a estos últimos.

En cuanto a las razones por las que prefieren las redes sociales, la mayoría de las respuestas apunta a la sencillez, a la simplicidad y a la interfaz más amigable que ofrecen las redes sociales. También conceden importancia al hecho de que Academia.edu o ResearchGate envían notificaciones o alertas cuando hay nuevos documentos relacionados con la temática de cada investigador. Consideran, en cambio, que los repositorios presentan más complejidad y exigen la introducción de más metadatos que las redes sociales por lo que el proceso de depósito es más largo.

También indican su preferencia por las redes sociales por el hecho de que consideran que tienen más alcance y más cobertura a nivel mundial que los repositorios.

Por último planteamos la pregunta de qué herramienta consideraban mejor para asegurar la preservación de las publicaciones a largo plazo. La sensación mayoritaria (72\%) es que es preferible depositar la documentación en un repositorio antes que en las redes sociales. Sorprende que teniendo tan clara esta cuestión sobre la importancia de la preservación de las publicaciones, en cambio, mayoritariamente, decidan utilizar más las redes sociales. 
Los resultados de la encuesta nos dejan constancia de que el grado de conocimiento de los repositorios, de su funcionamiento e incluso, en algunos casos, de su misma existencia, es muy bajo entre los profesionales. Para paliar esta percepción sobre los repositorios consideramos que es imprescindible que en las instituciones que alberguen alguno, institucional o temático, se lleven a cabo de manera regular talleres de formación para sus profesionales. Es importante que desde la institución se incentive a los autores para que utilicen más los repositorios. Para ello es importante promocionar el acceso abierto y sus beneficios entre los investigadores de la institución. Además es importante que estos organismos establezcan mandatos institucionales sobre la política de depósito, donde se explique al autor cómo debe depositar, qué tipos de documentos son aceptados, cuáles son los metadatos, etc. Los repositorios pueden proporcionar unos servicios de valor añadido como son estadísticas y métricas que ayudarán al autor a evaluar en cierta manera el impacto que ha tenido su producción científica.

Nuestra modesta recomendación es que los investigadores del patrimonio cultural depositen sus publicaciones en algún repositorio, ya que este, además de maximizar su visibilidad, también garantiza su preservación a largo plazo, aspecto de gran importancia y que las redes sociales no garantizan. Una vez depositada la publicación, se puede desde las redes sociales académicas. Por lo tanto, redes sociales y repositorios son compatibles, deben ser aliados en la estrategia de proporcionar difusión y visibilidad a los resultados de la investigación, pero siempre priorizando el depósito de los documentos en los repositorios.

\section{BIBLIOGRAFÍA}

- BORREGO, A. (2017) Institutional repositories versus ResearchGate: The depositing habits of Spanish researchers. Learned Publishing 30, 3; 2017, pp. 185-192 <https://doi. org/10.1002/leap.1099> [Consulta: 27/04/2020] 